\title{
Anterior Lobe of the Pituitary Gland
}

National Cancer Institute

\section{Source}

National Cancer Institute. Anterior Lobe of the Pituitary Gland. NCI Thesaurus. Code C12772.

The glandular or anterior lobe of the pituitary gland which secretes several hormones. 